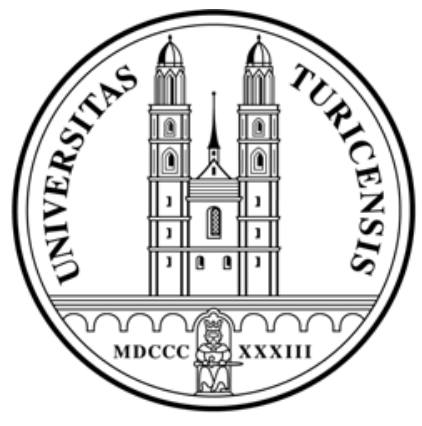

Institute for Empirical Research in Economics

University of Zurich

Working Paper Series

ISSN 1424-0459

Working Paper No. 223

\title{
An Application of Global Games to Signalling Models
}

Philipp C. Wichardt

July 2006 


\title{
An Application of Global Games to Signalling Models*
}

\author{
Philipp C. Wichardt ${ }^{\dagger}$ \\ Department of Economics, University of Bonn
}

This Version: July 8, 2006

\begin{abstract}
In a first attempt to apply the global games methodology to signalling games, Ewerhart and Wichardt (2004) analyse a beer-quiche type signalling game with additional imperfect information about the preferences of the receiver. Their approach allows them to dismiss the unreasonable pooling on quiche equilibrium. This paper revisits their example and discusses how an extension of the set of strategies for the sender affects the analysis. Interestingly, for an extended beer-quiche game, a unique equilibrium is selected while two equilibria are consistent with the Intuitive Criterion (Cho and Kreps, 1987). Apart from the technical analysis, potential economic applications of the results, e.g. in a context of limit pricing and entry deterrence, are indicated.
\end{abstract}

Key words: Global Games, Signalling, Equilibrium Selection, Intuitive Criterion, Limit Pricing

JEL code: C72, L11

*Acknowledgements: This paper was drafted during a visit at the Institute for Empirical Research in Economics at the University of Zürich. I am grateful to Christian Ewerhart for his invitation and his support. I am also grateful to Christian Ewerhart, Wolfgang Köhler and Georg Nöldeke for helpful comments and discussions.

${ }^{\dagger}$ Postal address for correspondence: Bonn Graduate School of Economics, Adenauerallee 24-42, D-53113 Bonn, Germany; e-mail: p.c.w@web.de. 


\section{Introduction}

In 1993, Carlsson and van Damme introduced the concept of global games as a means of equilibrium selection in normal form games. Inspired by the vast literature on this topic (e.g. Morris and Shin, 1998 and 2003), Ewerhart and Wichardt (2004) in an explorative study showed how the global games methodology as a means of equilibrium selection can be successfully applied to beer-quiche type signalling games. Their results suggest that the global games methodology may serve to support the Intuitive Criterion as proposed by Cho and Kreps (1987).

In this paper, we take up the suggested connection between global games and the Intuitive Criterion and show that already a slight extension of the message space of Player 1 (the sender) renders the global games approach more selective than the Intuitive Criterion. Consequently, any conjecture about a general equivalence of the two concepts for signalling games as suggested in Ewerhart and Wichardt has to be dismissed. Apart from the technical analysis of the extended beer-quiche game, we also discuss some economic applications of our results. In particular, we argue how the extended beer quiche game can be viewed as a simplified version of the Milgrom and Roberts (1982) model of limit pricing.

The rest of the paper is structured as follows. In Section 2, we briefly review the general line of argument followed by Ewerhart and Wichardt (henceforth $\mathrm{E} \& \mathrm{~W})$. Moreover, we provide some intuition for why an extension of the message space of the sender can render the global games approach to signalling games more selective than the Intuitive Criterion. In Section 3, we formally introduce the extended beer-quiche game as well as the technical details of the underlying model. Also in Section 3, we state and prove our main results. In Section 4, we briefly discuss the obtained results as well as their applicability to economic contexts. Section 5 concludes. 


\section{Global Beer-Quiche - a Review}

We begin our discourse with a brief review of the original beer-quiche game (Cho and Kreps, 1987). In this game, Player 1 (the sender) enters a bar to have his breakfast. He can be either strong or weak and his preferences are such that he prefers to have a beer if he is strong and to have a quiche if he is weak. The probability of Player 1 being strong is given by $p=0.9$. Unfortunately, at the bar Player 2 (the receiver) is waiting. He is in an aggressive mood and contemplating to fight the next guest to make an order. Nevertheless, he would only like to fight against someone who is weak. As the weak Player 1 prefers beer without fight to quiche with fight, each type of Player 1 ordering his respective preferred breakfast is not an equilibrium. Given such a strategy profile, a weak Player 1 would truly reveal his type and, hence, would have to face the fight. "Coordination" of the two types of Player 1 on either of the two messages would avoid this as Player 2 is assumed to be unwilling to fight the expected type of Player 1 . Yet, it appears to be somewhat surprising that in equilibrium a strong Player 1 should order a quiche. Cho and Kreps, therefore, used this example to introduce the Intuitive Criterion. The Intuitive Criterion requires that, in equilibrium, Player 2 at all unreached information sets assigns probability 0 to those types of Player 1 that could not actually benefit from a deviation to the respective message. In the beer quiche example this leads to the unique intuitive - prediction of pooling on beer.

Ewerhart and Wichardt (henceforth E\&W) show that the unique selection of pooling on beer can be replicated if a global games approach to the game is chosen. They introduce an additional type parameter $t$ for Player 2 that is only imperfectly known to Player 1 and that determines Player 2's payoff from fighting. In terms of the breakfast-in-the-bar story, we can think of Player 2 as being one of many possible types, all with different attitudes towards aggression. Player 1, then, entering the bar may look into the face of Player 
2 and, assuming that many fights will take their toll, this may reveal reliable information about the type of Player 2. Specifically, the type $t$ is drawn from a uniform distribution over $[-k,+k]$, where $k$ is chosen to be sufficiently large to make fighting strictly dominant/dominated in the respective extreme. The information Player 1 obtains about the type of Player 2 is modelled via a parameter $\widetilde{t}$ which in turn is uniform on an interval $\varepsilon$-close to the true realisation of $t$, i.e. $\tilde{t} \in[t-\varepsilon, t+\varepsilon]$. To avoid confusion, we will talk about $\tilde{t}$ as the signal Player 1 receives about the type of Player 2. The order Player 1 makes, i.e. beer or quiche, is referred to as his message to Player 2. The structure of the game as well as the payoffs are depicted in Figure 1.

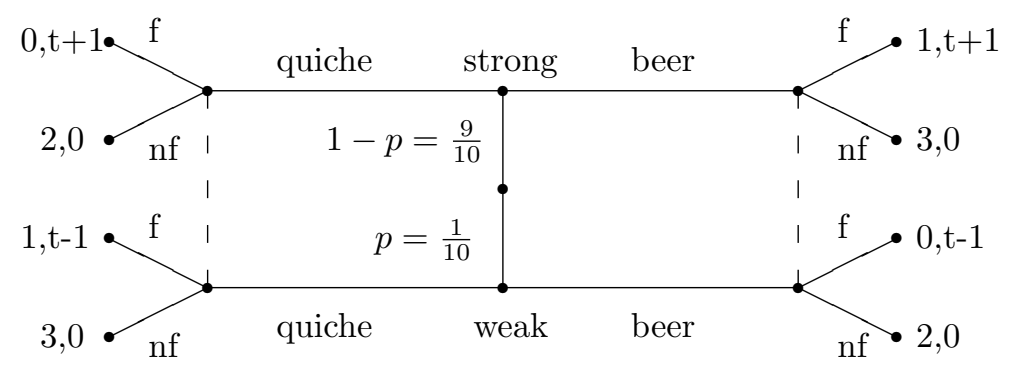

Figure 1: The global beer quiche game. $\mathrm{f}=$ fight, $\mathrm{nf}=$ not fight. The assumption is that Player 2 knows $t$; Player 1 only knows $\tilde{t} \in[t-\varepsilon, t+\varepsilon]$.

In E\&W the ensuing argument crucially hinges on the game's single crossing property: Given the signal $\widetilde{t}$, whenever the weak Player 1 has a weak incentive to play beer the strong Player 1 has a strict incentive to do so and whenever the strong Player 1 has a weak incentive to play quiche the weak Player 1 has a strict incentive to do so. This essentially ensures that the probability Player 2 has to assign to Player 1 being strong if he observes beer can never drop below the prior. Hence, it guarantees no fight against beer as long as Player 2 is not willing to fight against his prior, i.e. for all $t<0.8$, and implies that pooling on beer is the unique solution for all 
$\tilde{t} \in(-1,0.8-\varepsilon)$. As $\varepsilon$ converges to zero, this leads to the selection of a unique equilibrium almost everywhere (see E\&W, 2004, for the details). This equilibrium entails pooling on beer where desired.

In the sequel, we show how adding an additional message (xl-beer) to the beer-quiche game that is strictly dominated for the weak Player 1 and, hence, offers the strong Player 1 the option to unambiguously signal his type renders the global games approach more selective than the Intuitive Criterion. Roughly, the intuition is as follows. Assume that changing from beer to xlbeer (with no fight) is less costly for the strong Player 1 than is changing from beer to quiche (with fight) for the weak Player 1. Then, pooling on beer can never be part of an equilibrium for those signals $\widetilde{t}$ for which Player 1 can infer with certainty that Player 2 is willing to fight against his prior. The strong Player 1 would opt for xl-beer if his signal indicates that Player 2 is sufficiently aggressive to fight against his prior. The global games approach allows us to determine a unique switching point, i.e. a signal $\widetilde{t}$, for which the strong Player 1, in equilibrium, changes from beer to xl-beer. In the limit, i.e. if the signal is correct, this allows us to obtain a unique equilibrium prediction even for those cases where both pooling on beer as well as the strong Player 1 playing xl-beer and the weak Player 1 playing quiche survive the Intuitive Criterion in the underlying unperturbed game.

\section{The Extended Beer-Quiche Game}

In this Section, we analyse how the equilibrium of the global beer-quiche game (cf. Fig. 1) is affected if the set of signals available to the sender is extended by xl-beer, which is strictly dominated for the weak Player 1 . We begin our analysis with a description of the extended beer-quiche game. In a second step, we introduce the formal model used for the subsequent analysis. Finally, we state and prove the main result of this paper. 


\section{The Game}

For our analysis, we extend the global beer-quiche game introduced by E\&W adding a beer of size xl to the message space of the sender. By assumption xl-beer is strictly dominated for the weak Player 1 and gives 2.5 (if no fight) and 0.5 (if fight) to a strong Player 1 (see Figure 2).

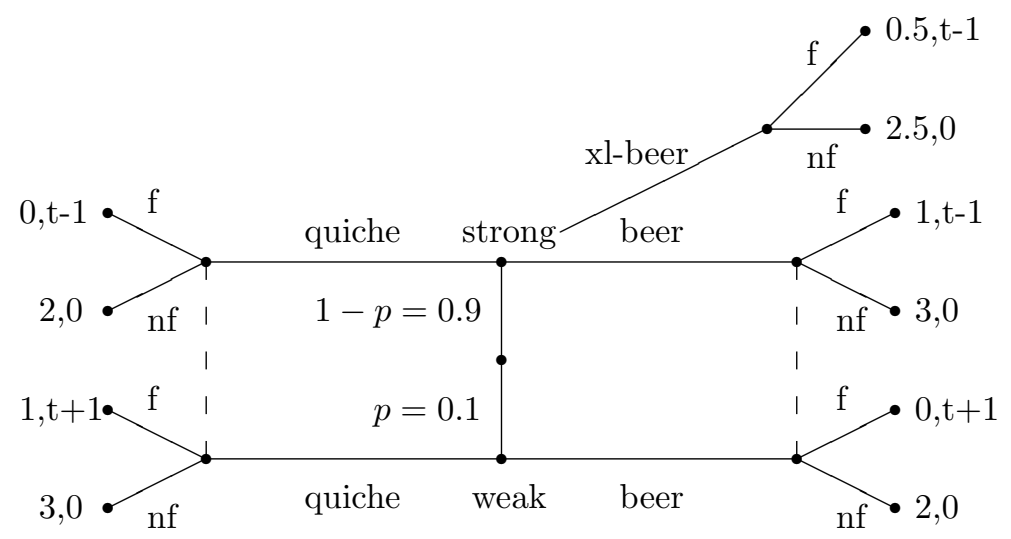

Figure 2: The extended global beer-quiche game. The dominated xl-beer for the weak type is omitted. Again Player 2 knows $t$; Player 1 only knows $\widetilde{t} \in[t-\varepsilon, t+\varepsilon]$.

Introducing xl-beer weakens the single crossing property used in E\&W as a weak incentive to play beer for the weak Player 1 no longer implies a strict incentive for the strong Player 1 to play beer. However, strictly speaking single crossing is still preserved. If we order the available messages for Player 1 in the obvious way, saying that xl-beer is stronger than beer which is stronger than quiche, single crossing can be stated as follows: taking Player 2 's actions as given, a change to a stronger message is always strictly better (less costly) for a strong Player 1 while changing to a weaker message is always strictly better (less costly) for a weak Player 1 . Moreover, the extended game (Figure 2) satisfies an additional property, referred to as $\mathrm{SC}+$, which will help us to establish uniqueness of the equilibrium. Specifically, it holds that, if 
Player 2 fights against quiche and does not fight against xl-beer, changing from beer to xl-beer is always strictly better (less costly) for the strong Player 1 than is changing from beer to quiche for the weak Player $1 .^{1} \mathrm{SC}+$ can be seen as an extension of single crossing. Single crossing implies that changing from beer to xl-beer is strictly better for the strong Player 1. However, xlbeer is strictly dominated by quiche for the weak Player 1. Consequently, he will never change to xl-beer but switch to quiche instead. Yet, if fight is certain against quiche, SC+ ensures that if the probability of fight against beer increases, the weak Player 1 switches from beer to quiche after the strong Player 1 switches from beer to xl-beer. Referring to both $\mathrm{SC}+$ and single crossing, we will say that the game satisfies an extended single crossing property.

\section{The Model (technical) ${ }^{2}$}

In order to present the formal framework needed for the ensuing analysis, we need to introduce some notation. Following E\&W, we denote the set of possible messages for Player 1 by $M$, i.e. $M=\{$ quiche, beer, $x l-b e e r\}$, and the set of possible responses for Player 2 by $R$, i.e. $R=\{$ fight, not fight $\}$. The set of types of Player 1 is denoted by $\Theta$, i.e. $\Theta=\{$ strong,weak $\}$, the set of possible types of Player 2 is denoted by $T$, i.e. $T=[-k, k]$ with $k>1$. Finally, the set of possible signals for Player 1 is denoted by $\widetilde{T}$, i.e. $\widetilde{T}=[-k-\varepsilon, k+\varepsilon] .{ }^{3}$ The resulting incomplete information game is denoted by $G(\varepsilon)$.

\footnotetext{
${ }^{1}$ Always means for all probabilities of fight against beer.

${ }^{2}$ We will use the concept of perfect Bayesian Equilibrium in distributional strategies. The reader who feels familiar with these concepts or is less interested in the technical details will be able to follow the main reasoning if he skips this subsection. Note only that, given $t, \widetilde{t}$ and message $m, \mu(m, t)$ denotes the probability Player 2 at $m$ assigns to Player 1 being strong, $\sigma_{1}(\widetilde{t}), \sigma_{2}(m, t)$ denote the Players' actions and $\sigma_{2}(a, m, t)$ denotes the probability with which Player 2 plays $a$.

${ }^{3}$ Different from E\&W, we do not treat the pair $(\theta, \widetilde{t})$ as the type of Player 1 . It seems easier to follow the argument if we make a clear distinction between the type $\theta$ and the signal $\widetilde{t}$ at this point.
} 
The course of events is the usual one. In the first stage, Nature determines a type and a signal $(\theta, \tilde{t}) \in \Theta \times \widetilde{T}$ for Player 1 , and a type $t \in T$ for Player 2. The pair $(t, \tilde{t})$ and $\theta$ are drawn independently. The probability distribution over $\Theta$ is denoted by $p($.$) , with p$ (strong $)=0.9$. We assume that $t$ is distributed uniformly over $T$ and that $\widetilde{t}$ is uniform on $[t-\varepsilon, t+\varepsilon]$. The respective conditional density functions for $\tilde{t}$ and $t$ are denoted by $\nu(. \mid t)$ and $\nu(. \mid \tilde{t})$. In the second stage, Player 1 observes his type and his signal and sends a message $m(\theta, \tilde{t}) \in M$. In the third stage, Player 2 observes his type $t$ and message send by Player 1 and chooses a response $r(m, t) \in R$. Finally, payoffs are determined by $u_{1}(m, r, \theta)$ for Player 1 , and by $u_{2}(r, \theta, t)$ for Player 2.

As was explained by E\&W, the players' strategies can be modelled by measurable functions

$$
\sigma_{1}: \Theta \times \widetilde{T} \rightarrow \Delta(M)
$$

for the Player 1 and

$$
\sigma_{2}: M \times T \rightarrow \Delta(R),
$$

for Player 2, where $\Delta(M)(\Delta(R))$ denotes the set of probability distributions over $M(R){ }^{4}$

Finally, Player 2, at each information set and for all types $t$, forms a belief about the type of Player 1 and the signal received by Player 1 . For all information sets $(m, t)$, we denote Player 2's belief that Player 1 is strong by $\mu(m, t)$. The belief about the signal received by Player 1 is determined by the conditional distribution $\nu(. \mid t)$.

\footnotetext{
${ }^{4}$ Technically, we apply the concept of distributional strategies (Milgrom and Weber, 1985), where a distributional strategy for Player $1 \theta, \theta \in \Theta$, is a probability distribution $\psi_{1 \theta}$ on $\widetilde{T} \times M$ such that the marginal distribution on $\widetilde{T}$ as induced by $\psi_{1 \theta}$ is identical to the marginal distribution of $\tilde{t}$ on $\widetilde{T}$; a distributional strategy for Player $2 m, m \in M$, is a probability distribution $\psi_{2 m}$ on $T \times R$ such that the marginal distribution on $T$ as induced by $\psi_{2 m}$ is identical to the marginal distribution of $t$ on $T$.
} 
The equilibrium concept employed is that of a perfect Bayesian Equilibrium (see Fudenberg and Tirole, 1991). Slightly abusing notation, we write $\sigma_{1}(m, \theta, \tilde{t})$ for Player 1 and $\sigma_{2}(r, m, t)$ for Player 2 to designate the probability weights placed on their respective actions given $\tilde{t}, t$.

Definition 1 A perfect Bayesian equilibrium (PBE) is comprised of a strategy profile $\sigma^{*}=\left(\sigma_{1}^{*}, \sigma_{2}^{*}\right)$ and beliefs $\mu(m, t)$ such that

1. for all $\left(m^{*}, \theta, \tilde{t}\right)$, if $\sigma_{1}^{*}\left(m^{*}, \theta, \tilde{t}\right)>0$ then

$$
m^{*} \in \arg \max _{m} \sum_{r} u_{1}(m, r, \theta) \operatorname{Pr}(r \mid m ; \tilde{t}),
$$

where

$$
\operatorname{Pr}(r \mid m ; \tilde{t}):=\int \sigma_{2}^{*}(r, m, t) d \nu(t \mid \tilde{t})
$$

2. for all $\left(r^{*}, m, t\right)$, if $\sigma_{2}^{*}\left(r^{*}, m, t\right)>0$, then

$$
r^{*} \in \arg \max _{r} \pi_{2}(r \mid m, t),
$$

where

$$
\pi_{2}(r \mid m, t):=u_{2}(r, \text { strong }, t) \mu(m, t)+u_{2}(r, \text { weak, } t)(1-\mu(m, t)) .
$$

3. for all $(\theta, m, t)$, if the information set of Player 2 corresponding to $m$ and $t$ is reached in equilibrium with strictly positive probability, i.e. if

$$
\sum_{\theta^{\prime}}\left[p\left(\theta^{\prime}\right) \cdot \int \sigma_{1}^{*}\left(m, \theta^{\prime}, \tilde{t}\right) d \nu(\tilde{t} \mid t)\right]>0
$$

then

$$
\mu(m, t)=\frac{p(\text { strong }) \cdot \int \sigma_{1}^{*}(m, \text { strong }, \tilde{t}) d \nu(\tilde{t} \mid t)}{\sum_{\theta}\left[p(\theta) \cdot \int \sigma_{1}^{*}(m, \theta, \tilde{t}) d \nu(\tilde{t} \mid t)\right]} .
$$




\section{Results}

As we will see, merely adding xl-beer leads to the selection of unique equilibrium actions for Player 1 in the (unperturbed) limit game $\widehat{G}(0)$, for almost all $\tilde{t}, t$. Despite the fact that in $\widehat{G}(\varepsilon)$ quiche can be dismissed for the strong type using iterated elimination of strictly dominated strategies, this result is somewhat surprising. For $\widehat{G}(0)$ and $\widetilde{t}, t \in(-1,0.8),{ }^{5}$ both pooling on beer as well as the strong Player 1 ordering xl-beer and the weak Player 1 ordering quiche are compatible with the Intuitive Criterion. ${ }^{6}$ Thus, in the present context, the global games approach is more selective than the Intuitive Criterion. The exact equilibrium strategies obtained using a global games approach to the extended beer-quiche game are given in Theorem 1.

To simplify notation, we introduce the following abbreviations: $\sigma_{s}(\widetilde{t})=$ $\sigma_{1}($ strong,$\widetilde{t}), \sigma_{w}(\widetilde{t})=\sigma_{1}($ weak,$\widetilde{t}), q=$ quiche, $b=$ beer, $x l=x l$-beer, $f=$ fight, $n f=$ no fight. Moreover, we denote the belief Player 1 holds about the probability with which Player 2 fights against message $m$, given $\widetilde{t}$, by $\eta(m \mid \widetilde{t})$. In equilibrium, $\eta(m, \widetilde{t})$ is determined by $\sigma_{2}$, i.e. the strategy of Player 2.

Theorem 1 In the limit, as $\varepsilon \longrightarrow 0$, equilibrium actions for both players are uniquely determined on the equilibrium path except for a set of measure zero. In particular, there exist $\underline{t}, t^{*}, \bar{t}$ such that in the limit we get:

\footnotetext{
${ }^{5}$ In this case, i.e. with $\varepsilon=0$, we have $t=\widetilde{t}$.

${ }^{6}$ Pooling on beer is obviously compatible with the intuitive criterion. To support fight against beer in the separating equilibrium, Player 2 has to assign a high probability to weak types at his beer information set. This is possible under the intuitive criterion since both types of Player 1 may potentially profit from a deviation to beer and thus off path beliefs against beer are not subject to any restrictions.
} 


$$
\begin{gathered}
\sigma_{s}(\widetilde{t})=\left\{\begin{array}{cc}
b & \text { if } \tilde{t} \in \widetilde{T} \backslash\left[t^{*}, \bar{t}\right] \\
x l & \text { if } \tilde{t} \in\left(t^{*}, \bar{t}\right)
\end{array}, \sigma_{w}(\widetilde{t})=\left\{\begin{array}{rr}
q & \text { if } \tilde{t} \in \widetilde{T} \backslash\left[t, t^{*}\right] \cup\{\bar{t}\} \\
b & \text { if } \tilde{t} \in\left(\underline{t}, t^{*}\right)
\end{array}\right.\right. \\
\sigma_{2}(f, q, t)=\left\{\begin{array}{ll}
0 & \text { if } t<\underline{t} \\
1 & \text { if } t>\underline{t}
\end{array}, \quad \sigma_{2}(f, b, t)=\left\{\begin{array}{cc}
0 & \text { if } t<t^{*} \\
\geq 0.5 & \text { if } t \in\left(t^{*}, \bar{t}\right) \\
1 & \text { if } t>\bar{t}
\end{array}\right.\right. \\
\sigma_{2}(f, x l, t)=\left\{\begin{array}{ll}
0 & \text { if } t<\bar{t} \\
1 & \text { if } t>\bar{t}
\end{array} .\right.
\end{gathered}
$$

Strategies are undetermined where not specified, i.e. at the switching points.

Remark 1 For our particular example we obtain: $\underline{t}=-1, t^{*}=\frac{7}{11}$ and $\bar{t}=1$. See Figure 3 for illustration.
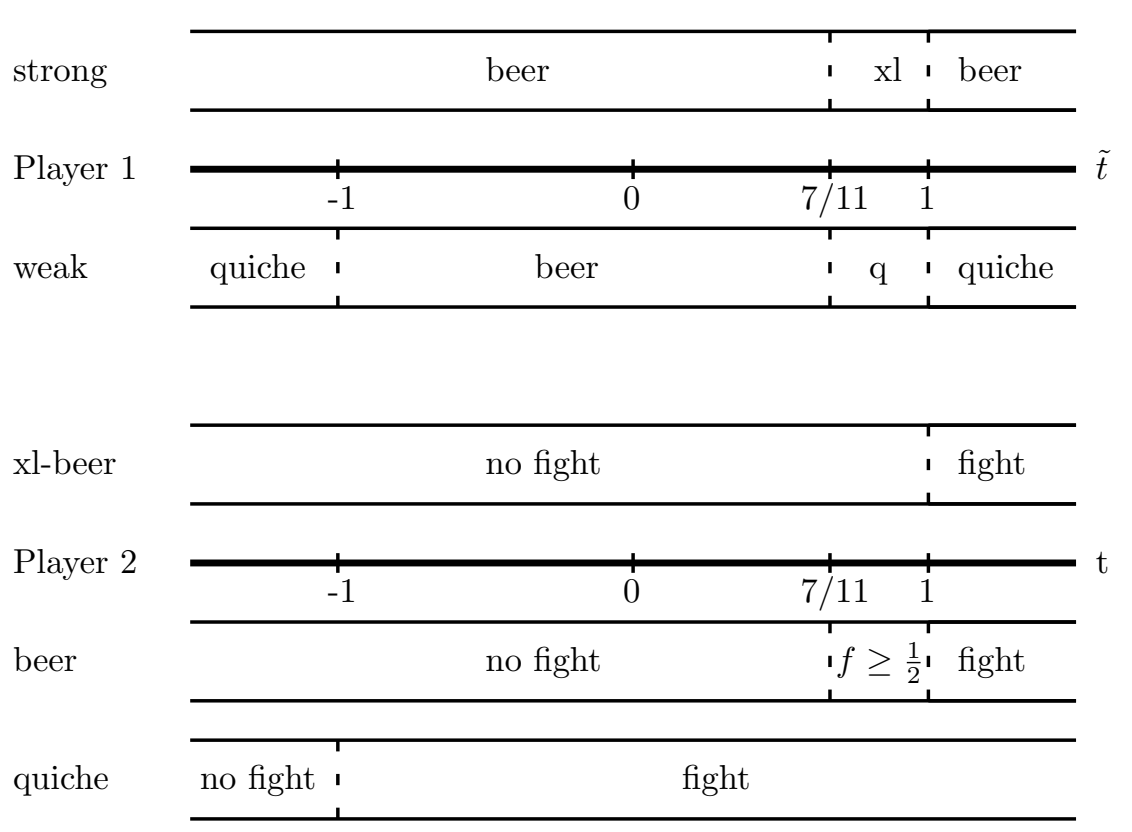

Figure 3: Equilibrium Strategies for $\varepsilon \rightarrow 0$ as stated in Theorem 1. 
Corollary 1 In the present context, the global games approach is stronger than the Intuitive Criterion.

Before moving on to the formal derivation of Theorem 1, we first provide a sketch of the proof. To prove Theorem 1 , we consider $\widehat{G}(\varepsilon)$ for $\varepsilon>0$. The argument, which is subdivided into 4 lemmas, is structured as follows. After some preparatory (iterated) dominance arguments (establishing mainly $\sigma_{s}=b, \sigma_{w}=q$ for $|\widetilde{t}|>1$ ), we first show that as $\tilde{t}$ gets larger than -1 , i.e. as quiche is no longer dominant for the weak Player 1 (using two steps of iterated dominance), we get $\sigma_{w}(\widetilde{t})=\sigma_{s}(\widetilde{t})=b$ for some range of signals $\tilde{t}$ (Lemma 1). - This part of the argument is similar to the standard global games argument. - Next, we employ additional equilibrium conditions in conjunction with the extended single crossing property and show that, if in equilibrium there is a $t^{*}$ such that at $\widetilde{t}=t^{*}$ the strong Player 1 changes from beer to $x$ l-beer, then $t^{*}$ is uniquely determined, given $\varepsilon$ (Lemma 2). In equilibrium, this change, which necessarily is accompanied by the weak Player 1 changing back to quiche, has to occur at some point (Lemma 3). It follows that, if there exists an equilibrium for this game, then the strategies of Player 1 and Player 2 are already uniquely determined for all $t, \tilde{t}$ except for a set of measure proportional to $\varepsilon$. Finally, we show how the remaining gaps can be filled to establish existence of the equilibrium (Lemma 4). Putting things together and letting $\varepsilon$ converge to zero, we obtain Theorem 1.

Proof of Theorem 1. To begin with, we specify the regions for Players in which one of their actions is strictly dominant (or a strict best response using iterated elimination of strictly dominated strategies). For Player 2 $\sigma_{2}(t)=n f$ is strictly dominant for $t<-1 ; \sigma_{2}(t)=n f$ is dominant against the ex ante expected type of Player 1 for $t<0.8$; and $\sigma_{2}(t)=f$ is strictly dominant for $t>1$. Moreover, $\sigma_{2}(t)=n f$ is a strict best response for Player 2 against $x l$-beer for all $t<1$ since this message is strictly dominated for the weak Player 1. Further iterating the dominance argument, we obtain 
$\sigma_{s}(\widetilde{t})=b$ and $\sigma_{w}(\widetilde{t})=q$ for $|\widetilde{t}|>1+\varepsilon$, and $\sigma_{s}(\widetilde{t}) \neq q$ for all $t(x l$ or $b$ always make the strong Player 1 better off). Finally, it follows that $\sigma_{2}(t)=f$ against $q$ for all $t>-1$ (as by the previous argument $\mu(q, t)=0$ ).

After this preparatory work, we proceed with the main step of the proof, which is divided into four Lemmas.

Lemma 1 In equilibrium, $\sigma_{s}(\widetilde{t})=\sigma_{w}(\widetilde{t})=b$, for $\widetilde{t} \in\left(-1, \frac{5}{13}-\varepsilon\right)$ and $\varepsilon$ small.

Proof. From the above dominance argument, we know that $\sigma_{2}(b, t)=n f$ for all $t<-1$. Assume that the smallest value of $t$ for which Player 2 in equilibrium is indifferent against beer is given by $t^{\prime}>-1$. Then, $\sigma_{s}(\widetilde{t})=b$ is optimal for the strong Player 1 at least for all $\widetilde{t}<t^{\prime}-\varepsilon$. Moreover, the strong Player 1 will only switch from beer to $x l$-beer if $\eta(b, \widetilde{t}) \geq 0.25$. Otherwise, beer gives the higher expected payoff. Hence, in equilibrium we have $\sigma_{s}(\widetilde{t})=b$ at least for all $\widetilde{t}<t^{\prime}-\frac{\varepsilon}{2}$. It follows that Player 2 being of type $t^{\prime}$ has to assign at least probability 0.25 to the strong Player 1 playing beer. Consequently, in equilibrium, for $t=t^{\prime}$, Player 2's belief against beer is bounded. He at least has to assign probability 0.25 to strong types ordering beer and at most can assign probability 1 to weak types doing so, i.e.

$$
\mu\left(b, t^{\prime}\right) \geq \frac{0.25 \cdot 0.9}{0.1+0.25 \cdot 0.9}=\frac{9}{13} .
$$

Therefore, no fight remains the strict best response against beer at least for all $t$ with

$$
\frac{4}{13}(t+1)+\frac{9}{13}(t-1)<0
$$

i.e. for all

$$
t<\frac{5}{13}
$$

This implies that $\frac{5}{13}$ is a lower bound for $t^{\prime}$, so that $\sigma_{s}(\widetilde{t})=b$ at least for all 
$\widetilde{t} \in\left(-1, \frac{5}{13}-\varepsilon\right)$. As $\sigma_{2}(t)=f$ against quiche for all $t>-1,{ }^{7}$ it follows that also $\sigma_{w}(\widetilde{t})=b$ at least for all $\widetilde{t} \in\left(-1, \frac{5}{13}-\varepsilon\right)$.

Lemma 2 If in equilibrium the strong Player 1 changes from beer to xl-beer, then this change has to occur for $\widetilde{t}=\frac{7}{11}-\frac{\varepsilon}{2}$. Moreover, in this case Player 2 changes from no fight against beer to fight at $t=\frac{7}{11}$ and the weak Player 1 changes back to quiche at $\widetilde{t}=\frac{7}{11}$.

Proof. Again let $t^{\prime}$ be the smallest value of $t$ for which Player 2 is indifferent against beer. Then, $\sigma_{s}(\widetilde{t})=b$ at least for $\tilde{t} \in\left[t^{\prime}-\varepsilon, t^{\prime}-\frac{\varepsilon}{2}\right.$ ) (as was shown in the proof of Lemma 1). Moreover, from Lemma 1 we know that $\sigma_{s}=\sigma_{w}=b$ for some range of $\tilde{t}>-1$. As for $\widetilde{t}>-1+\varepsilon$ we have $\eta(q, \widetilde{t})=1,{ }^{7}$ a weak Player 1 will change back from beer to quiche only if $\eta(b, \widetilde{t}) \geq 0.5$. Thus, we can infer that $\sigma_{s}(\widetilde{t})=\sigma_{w}(\widetilde{t})=b$ at least for all $\widetilde{t} \in\left[t^{\prime}-\varepsilon, t^{\prime}-\frac{\varepsilon}{2}\right)$.

Now, for all $\tilde{t} \in(-1,1-\varepsilon)$ it holds that whenever the strong Player 1 has an incentive to play beer, the weak Player 1 has a strict incentive to do so (a consequence of the extended single crossing property). It follows that whatever Player 2's beliefs at $t^{\prime}$ are, $\mu(b, t)$ cannot increase for all $t \in$ $\left(t^{\prime}, t^{\prime}+\frac{\varepsilon}{2}\right)$. Yet, as $t$ increases the willingness of Player 2 to fight increases. Therefore, $\sigma_{2}(t)=f$ for all $t \in\left(t^{\prime}, t^{\prime}+\frac{\varepsilon}{2}+\gamma\right)$, for some $\gamma \geq 0$. Knowing that Player 2 will fight against beer for all $t \in\left(t^{\prime}, t^{\prime}+\frac{\varepsilon}{2}+\gamma\right)$ the strong type of Player 1 strictly prefers to play $x l$-beer for all $\tilde{t} \in\left(t^{\prime}-\frac{\varepsilon}{2}, t^{\prime}+\varepsilon+\gamma\right)$. Repeating the preceding argument, we can conclude that the change of the strong type to $x$ l-beer has to last at least as long as the beer information set for Player 2 remains reached. This implies that Player 2's belief at $t^{\prime}$ is determined by the respective probabilities of fight against beer needed to induce a switch from beer to xl-beer (quiche) of the strong (weak) type of Player 1. To be

\footnotetext{
${ }^{7}$ See the argument preceding Lemma 1.
} 
precise, Player 2's belief are given by

$$
\mu\left(b, t^{\prime}\right)=\frac{0.25 \cdot 0.9}{0.25 \cdot 0.9+0.5 \cdot 0.1}=\frac{9}{11} .
$$

Since Player 2 is assumed to be indifferent at $t^{\prime}$, and in fact has to be if a change in his strategy is to occur at $t^{\prime}$, this determines a unique $t^{\prime}$ for which this change indeed is possible:

$$
\begin{aligned}
\left(1+t^{\prime}\right) \frac{2}{11}+\left(-1+t^{\prime}\right) \frac{9}{11} & =0 \\
t^{\prime} & =\frac{7}{11} .
\end{aligned}
$$

If, in equilibrium, for some $t>t^{\prime}$ the beer information set of Player 2 was reached again, the relative proportions would have to be at least as the above and, thus, fight would have to be the unique possible response in equilibrium. Hence, if a change in Player 1's behaviour away from beer occurs, it has to persist at least for all $\widetilde{t}<1-2 \varepsilon$. This proves Lemma 2 ).

Lemma 3 In equilibrium, $\sigma_{s}(\widetilde{t})=x l$ for $\widetilde{t} \in(0.8+\varepsilon, 1-2 \varepsilon)$.

Proof. Consider the case where Player 1 gets a signal $\tilde{t} \in(0.8+\varepsilon, 1-2 \varepsilon)$. As mentioned in the proof of Lemma 2 , if for a given signal $\widetilde{t} \in(0.8+\varepsilon, 1-2 \varepsilon)$ the strong type prefers to play beer instead of $x$-beer, the weak type will certainly prefer beer to quiche. Thus, the probability Player 2 has to assign to Player 1 being weak if his beer information set is reached for some $t \in$ $(0.8+2 \varepsilon, 1-3 \varepsilon)$ is bounded below by the prior. Consequently, the only possible response to beer is fight. Hence, only the separating equilibrium in which $x l$-beer is ordered by strong types and quiche is ordered by weak types can actually be played. The switch from $\sigma_{s}(\widetilde{t})=\sigma_{w}(\widetilde{t})=b$ to $\sigma_{s}(\widetilde{t})=x l$-beer and $\sigma_{w}(\widetilde{t})=q$, therefore, has to occur at some point. As argued above, this point is uniquely determined by the indifference condition (cf. Lemma 2). 
Lemma 4 For \& small, we can specify actions for both Player 1 and Player 2 for all remaining $t, \tilde{t}$ such that together with the above described actions we obtain a perfect Bayesian equilibrium of the extended global beer-quiche game.

Proof. What remains to be shown is how equilibrium actions look like around $t, \tilde{t}=1$. The potential trouble in this region derives from the fact that it is not optimal for the strong Player 1 to change his strategy from beer to $x l$-beer immediately as $\tilde{t}$ drops below $1+\varepsilon$. As a consequence of this, the beer information set of Player 2, in equilibrium, remains reached for all $t \in(1-\rho, 1)$, for some $\rho>0$. Hence, beliefs at the beer information set have to be determined by Bayes' Rule, for $t \in(1-\rho, 1)$, and as long as the proportion of strong types is large enough, no fight is the optimal response. This in turn extends the range of signals for which it is optimal for the strong type to play beer, and so forth. Yet, to make the actions derived so far part of an equilibrium, Player 2 has to start playing fight somewhere close to $t=1$. In order to achieve this is, however, the weak Player 1 has to play beer for some $\tilde{t} \in(1-2 \varepsilon, 1-2 \varepsilon)$, and Player 2 in turn has to choose no fight against beer sufficiently long to make the weak Player 1 willing to do so; in particular, we need $\sigma_{2}(b, t)=n f$ for all $t \in(1-\varepsilon, 1)$. The respective fraction of weak types, who will be indifferent, then has to choose actions so as to induce exactly the beliefs necessary to support a switching back of Player 2 to fight as $t<1-\varepsilon .^{8}$ In that case, the strong type switches to xl-beer as fight against beer becomes sufficiently likely. In the present example, this is the case for $\tilde{t}<1-\frac{\varepsilon}{2}$.

\footnotetext{
${ }^{8}$ Notice that whenever it is better for the weak Player 1 to try to imitate the strong type, i.e. whenever the profit to the weak type from beer with no fight is larger than the profit from quiche with fight, there has to be some $\widehat{\varepsilon}<2 \varepsilon$ such that no fight against beer for all $t \in(1-\widehat{\varepsilon}, 1)$ and fight otherwise makes all weak types of Player 1 getting a signal $\widetilde{t} \in(1-\varepsilon, 1+\varepsilon-\widehat{\varepsilon})$ indifferent between quiche and beer. For $\varepsilon$ small enough this fraction of weak types suffices to induce the necessary switch of Player 2 to fight against beer as $t<1-\widehat{\varepsilon}$.
} 
To ensure that the small fraction of weak types indeed suffices to induce the necessary beliefs although the willingness to fight decreases as $t$ decreases, $\varepsilon$ has to be sufficiently small. If $\varepsilon$ is small, it suffices that $\sigma_{w}(\widetilde{t})=q$ for $\widetilde{t}>1-\frac{\varepsilon}{2}$, but $\sigma_{w}(\widetilde{t})=b$ for $\widetilde{t} \in(1-\varepsilon, 1-\varepsilon+\omega), 0<\omega=\omega(\varepsilon)<\frac{\varepsilon}{2}$, to induce the necessary beliefs. Given his type $t$, the expected payoff $\Pi$ of Player 2 is given by

$$
\begin{aligned}
u_{2}(f \mid b, t) & =(t+1) \cdot(1-\mu(b, t))+(t-1) \cdot \mu(b, t) \\
& =t-1+2 \cdot(1-\mu(b, t)) .
\end{aligned}
$$

For $\varepsilon$ small we know that with the above proposed strategies $\mu(b, t)$ for $t=$ $1-\varepsilon$ are given by

$$
\mu(b, t)=\frac{\frac{\varepsilon / 2}{2 \varepsilon} \cdot 0.9}{\frac{\omega}{2 \varepsilon} \cdot 0.1+\frac{\varepsilon / 2}{2 \varepsilon} \cdot 0.9} .
$$

As $t$ falls below $1-\varepsilon$ this probability decreases nonlinearly since only the strong types are cut out but the fraction of weak types playing beer is unaffected. Hence, since $t$ decreases, the increase in $u_{2}(f \mid b, t)$ due to the increase in $1-\mu(b, t)$ dominates the direct effect of $t$ on $u_{2}(f \mid b, t)$. Consequently, changing from no fight to fight indeed is possible. This proves Lemma 4 and, thereby, completes the proof of Theorem 1. qed.

Let us briefly recap the crucial features of the extended beer-quiche game that lead to Theorem 1. On the one hand, we obviously exploit the fact that $x l$-beer is strictly dominated for the weak Player 1 . On the other hand, we used the extended single crossing property to restrict the possible beliefs of Player 2. The fact that, as the probability of fight against beer increases, the strong Player 1 gets an incentive to switch to $x$-beer before the weak Player 1 gets an incentive to return to quiche guarantees a unique equilibrium. Without it, equilibria as in E\&W will occur. ${ }^{9}$ To facilitate the argument, we

\footnotetext{
${ }^{9}$ In this kind of equilibrium, the strong Player 1 stays with beer for all $\tilde{t} \in(0.8,1)$, the weak Player 1 changes between beer and quiche in a way that keeps Player 2 indifferent
} 
chose the payoffs from xl-beer to be such that quiche for the strong Player 1 can be eliminated by iterated dominance. Notice however, that as long as the extended single crossing property is satisfied (and xl-beer is not dominated for the strong Player 1), Theorem 1 continues to hold. Single Crossing guarantees that the strong Player 1 never plays quiche.

\section{Discussion}

As we have seen in Section 3, extending the list of possible messages by xl-beer simplifies the analysis of the global beer-quiche game substantially. Without invoking further existence results, as was necessary in E\&W, we were able to specify equilibrium actions for the global signalling game. In the limit, we obtained equilibrium actions for both Players that are unique for almost all $t, \tilde{t}$. In contrast to this, two equilibria survive the Intuitive Criterion for the underlying unperturbed game with $t, \tilde{t} \in(-1,0.8), \varepsilon=0$.

The reason for this discrepancy is that the Intuitive Criterion only requires off path beliefs of the receiver to place zero probability on all those types of the sender that would never profit from a deviation to the respective message in the equilibrium under consideration. Otherwise beliefs are not restricted. Thus, for the current example, Player 2 is "free" to assign a high probability to the weak Player 1 against beer and to assume the strong Player 1 to play $x l$-beer already for $t=-0.99$ (thus sustaining the separating equilibrium with $\sigma_{s}=x l$ and $\sigma_{w}=q$ ), or to assume both types of Player 1 to pool on beer for $t=0.79$. The noisy information structure of the global game (i.e. for $\varepsilon>0$ ) excludes these possibilities, as it necessitates the incorporation of expected behaviour in adjacent regions into the players' analysis. Yet, not

against beer. Player 2 in turn "mixes" against beer (i.e. changes between fight and not fight) in such a way that the weak Player 1's behaviour is optimal. Existence of such an equilibrium was proved in E\&W. However, statements about convergence of actions for $t, \widetilde{t} \in(0.8,1)$ as $\varepsilon \longrightarrow 0$ appear difficult for this case. 
only do outer dominance regions influence the players' beliefs (e.g. making it optimal for a strong Player one to stay with beer around $\widetilde{t}=-1$ ). Equilibrium requirements for the whole game in conjunction with the extended single crossing property also allow us to further restrict Player 2's beliefs (in the sense that, if a certain information set was reached, Player 2's belief at this information set would have to be in accord with single crossing). Thus, taking limits $(\varepsilon \rightarrow 0)$, we obtain tighter restrictions on Player 2's beliefs as compared to case where the Intuitive Criterion is applied to the underlying unperturbed game.

Following the above discussion, a natural question that arises is how further extensions of the set of possible messages for Player 1 will affect the outcome. In particular, it would be interesting to see how how the outcome is affected by intermediate messages, e.g. between beer and $x l$-beer, that tie in with the extended single crossing but are not dominated for the weak Player 1. Unfortunately, this question in general is difficult to answer. Of course, the equilibrium described in Theorem 1 will be preserved as we can always assume all of the new messages to be responded by fight whenever this is not strictly dominated for Player 2, i.e. for $t>-1$ (in this case, they will never be chosen). Yet, it is not clear whether uniqueness still obtains.

Another point that deserves a mention is the special structure of the payoff perturbation. Assuming incomplete information of Player 1 about Player 2's payoff only from fighting may appear to be rather restrictive. For signalling games in which the payoff of the receiver solely depends on the type of the sender and not on the actual message sent, however, perturbing the receiver's payoffs from one response only is fairly comprehensive. What matters to Player 1 is how much the receiver values one action relative to the other. For this our approach allows us to reflect the whole variety of possible valuations. Manipulating one payoff suffices to introduce incomplete information about the characteristic of Player 2 that is relevant for the game. 
Finally, we want to emphasise the economic applicability of our model. The extended beer-quiche game discussed in Section 3 can, for example, be viewed as a simplified version of the Milgrom and Roberts (1982) model of limit pricing and entry deterrence. ${ }^{10}$ In this case, we would relate the type parameter of Player 2, the entrant, to the fixed cost of entry. The type of Player 1, the incumbent, refers to his cost of production. A strong incumbent has low production costs whereas a weak incumbent has high costs of production. Beer and quiche are the respective monopoly quantities of the incumbent and xl-beer would be an output the weak incumbent would never want to produce. The unique equilibrium we obtain then can be interpreted in the following way: if entry costs are low (high), the entrant always (never) enters the market in the second period and therefore the incumbent produces his respective monopoly output in the first round; between the two extremes, both incumbent types pool on the monopoly output of the low cost type and no entry occurs if entry costs are rather high; if entry costs are intermediate, the low cost incumbent successfully limit prices and the high cost incumbent concedes. If only three possible outputs are considered, the equilibrium is unique for almost all entry costs. ${ }^{11}$

\section{Concluding Remarks}

Building on the findings of E\&W (2004) we further pursued the question how and under which circumstances a global games approach to signalling games will be fruitful and how it compares to the Intuitive Criterion. For the extended beer-quiche game analysed in this paper it turned out that through the global games approach we are able to discriminate between two equilibria which are compatible with the Intuitive Criterion. Thus, we concluded that

\footnotetext{
${ }^{10}$ See also Bain, 1949, and Matthews and Mirman, 1983.

${ }^{11}$ Another potential example is a central bank signalling context where the central bank (Player 1) is imperfectly informed about the traders (Player 2) potential alternative investments.
} 
in general both concepts will not be equivalent. Furthermore, we briefly discussed both the approach taken and the obtained results and indicated some interesting economic applications.

The global games methodology in general is very appealing as it introduces incomplete information about the payoffs of the game in a way that appears to be very natural. A better understanding of its impact on the analysis also of signalling games, therefore, seems to be desirable. We hope that the preceding discussion has helped the reader to gain a better picture of the analytical value of a global games approach also to signalling games.

\section{References}

Bain, J., 1949, "A Note on Pricing in Monopoly and Oligopoly," American Economic Review 39, pp.448-464.

Carlsson, H. and E. van Damme, 1993, "Global Games and Equilibrium Selection," Econometrica 61, pp. 989-1018.

Cho, I.-K. and D. M. Kreps, 1987, "Signalling Games and Stable Equilibria," Quarterly Journal of Economics 102, pp. 179-221.

Ewerhart, C. and P. Wichardt, 2004, "Signalling, Globality and the Intuitive Criterion," Working Paper 189, Institute for Empirical Research in Economics, University of Zürich.

Fudenberg, D. and J. Tirole, 1991, Game Theory, MIT Press, Cambridge, Massachusetts.

Matthews, S. and L. Mirman, 1983, "Equilibrium Limit Pricing: The Effects of Private Information and Stochastic Demand," Econometrica 51, pp. 981996. 
Milgrom, P. and R. Weber, 1985, "Distributional Strategies for Games with Incomplete Information," Mathematics of Operations Research 10, pp. 619631.

Milgrom, P. and J. Roberts, 1982, "Limit Pricing and Entry Under Incomplete Information: An Equilibrium Analysis," Econometrica 50, pp. 443459.

Morris, S. and H. S. Shin, 1998, "Unique Equilibrium in a Model of SelfFulfilling Currency Attacks," American Economic Review 88, pp. 587-597.

Morris, S. and H. S. Shin, 2003, "Global Games: Theory and Applications," in: Dewatripont, M., L. Hansen, and S. Turnovsky (eds.), Advances in Economics and Econometrics, the Eighth World Congress, Cambridge, UK, in press.

van Damme, E., 1991, Stability and Perfection of Nash Equilibria, Springer, 2nd edition. 